\title{
Nonlinear Analysis of Wiggler Taper, Mode Competition, and Space-Charge Effects For a 280-GHz Free Electron Laser
}

\author{
SHENG-FUH R. CHANG, JURIANTO JOE, STUdent member, IEeE, AND JOHN E. SCHARER
}

\begin{abstract}
The nonlinear characteristics of a $280-\mathbf{G H z}$ free electron laser (FEL) are simulated by numerical computation. The threedimensional set of coupled nonlinear differential equations is solved for a set of TE and TM modes valid for the high gain Compton regime. The use of a nonlinear taper for efficiency enhancement, the sensitivity of gain to competing mode power levels, space-charge effects, and the effect of electron beam source distributions on gain and efficiency are examined for a 10-MeV 3-kA beam. It is found that the nonlinear taper greatly enhances the gain and efficiency and makes the saturation power levels relatively insensitive to the competing mode power levels. The efficiency is increased to $48 \%$ by means of a nonlinear taper in which the 3-D and wiggler-averaged codes are compared and the effects of space charge are found to reduce the efficiency to a level of $32 \%$. The effect of beam quality in terms of the four volume phase space is examined and found to have an observable effect at this wavelength.
\end{abstract}

\section{INTRODUCTION}

$\mathrm{T}$ HE FREE ELECTRON laser has been shown [1] to operate quite effectively at high powers and efficiency in the $35-\mathrm{GHz}$ microwave range. The scaling of these results to the millimeter-wave range at $280 \mathrm{GHz}$ is currently underway [2], [3], and applications to the areas of communications, high resolution radar, spectroscopy, and the heating of fusion plasmas are envisioned. Work by Ganguly and Freund [4], [5] and Freund et al. [6], [7] has formulated the three-dimensional analysis and set of coupled nonlinear differential equations which self-consistently solve for the ensemble of electrons and mode wave fields in a circular or rectangular waveguide. Beam-self and space-charge fields are neglected in the analysis so that it is valid for the high-gain Compton regime. We previously modified this analysis and examined computational solutions for a helical quadrupole wiggler [8], [9] operating at 35 and $300 \mathrm{GHz}$. Ganguly and Freund [10] recently utilized an approximate method which expands the space-charge field in terms of the Trivelpiece-Gould modes for a fully filled cylindrical waveguide. This method is found to accurately model experiments in which the beam fills most of the guide radius and the case of

Manuscript received September 25, 1989; revised January 16, 1990 This work was supported by the University of Wisconsin Graduate School and the National Science Foundation under Grant ECS-8514978.

The authors are with the Electrical and Computer Engineering Department, University of Wisconsin, Madison, WI 53706.

IEEE Log Number 9035362. near grazing intersection between the beam line and waveguide dispersion curves.

In this paper, we examine the effect of a nonlinear wiggler taper and the sensitivity of gain to competing mode power levels, we compare the 3 -D solutions to a wiggleraveraged solution including space-charge forces, and we investigate the effect of electron beam source distributions on the gain and efficiency for a $280-\mathrm{GHz}$ FEL. The anal$y$ sis and system of equations for the Compton regime are similar to that derived by Freund et al. [6], [7]. In this paper, we examine the effect of a nonlinear taper and electron source distributions on FEL gain and efficiency. For the nonlinear taper, Kroll et al. [11] (abbreviated as KMR henceforth) and Cover et al. [12] examined its effect on a single mode; we extend this to investigate its effect for the other competing modes, since a highly overmoded waveguide is usually employed for guiding radiation at millimeter wavelengths. Goldstein et al. [13] have examined the effect of electron beam source distribution in the optical regime and found that there is a factor of ten difference in the small-signal gain of the FEL oscillator for different distributions. Colson and Blau [14] and Boyd et al. [15] have examined phase space source distributions and their effect on gain. For the millimeter-wave FEL amplifier of interest, we look at effects from the small-signal to the saturation regimes.

In Section II, the set of governing equations is derived including simultaneously interacting waveguide modes, the hypervolume phase space distribution for an electron beam, and the prescription for the optimum taper rate. Section III describes the numerical solution for a $280-\mathrm{GHz}$ case in which the nonlinear taper, mode competition, a comparison with a wiggler averaged code, and space charge effects are considered. The effects of a Gaussian and uniform-source phase space representation on the gain and efficiency are examined as well.

\section{Analysis of the Interaction Dynamics}

The schematic configuration of an FEL amplifier with a linear dipole wiggler is shown in Fig. 1. The relativistic electron beam is described as in accelerator physics by a distribution function in phase space. Assuming that the electron beam is monoenergetic and that none of the electrons intersect the wall, the current density can be written 


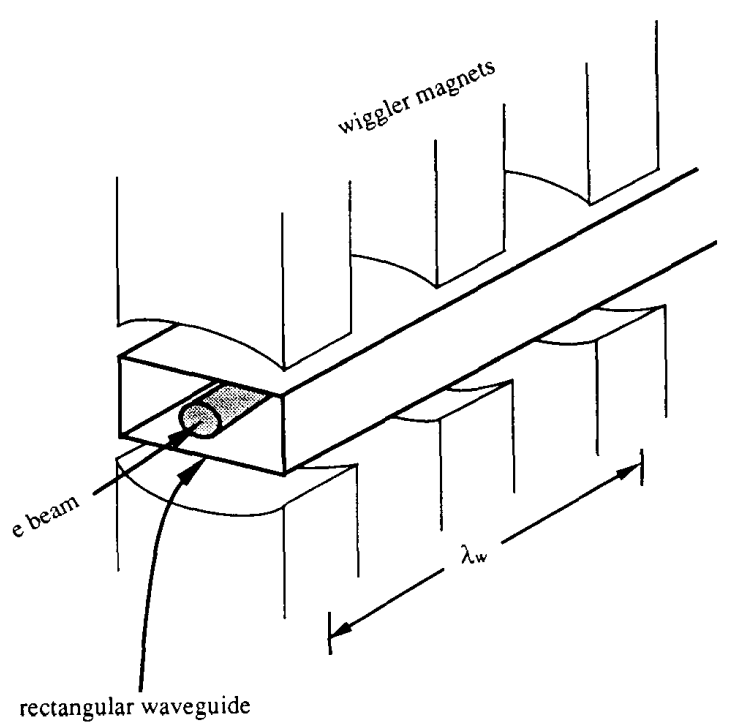

Fig. 1. Schematic view of FEL amplifier with dipole wiggler contiguration.

as follows:

$$
\begin{aligned}
\boldsymbol{J}(\boldsymbol{x}, t)= & -e n_{b} \iiint_{\Omega_{\perp}} \int d p_{x o} d p_{y^{o}} d x_{o} d y_{o} \\
& \cdot D\left(\boldsymbol{p}_{\perp}^{o}, \boldsymbol{x}_{\perp}^{o}\right) v_{-} \int_{-T / 2}^{T / 2} d t_{o} \sigma_{\|}\left(t_{o}\right) \\
& \times v\left(z ; \boldsymbol{p}_{\perp}^{o}, \boldsymbol{x}_{\perp}^{o}, t_{o}\right) \delta^{2}\left(\boldsymbol{x}_{\perp}-\xi_{\perp}\right) \frac{\delta(t-\tau)}{\left|v_{z}\right|}
\end{aligned}
$$

with

$$
\begin{aligned}
& \tau\left(z ; \boldsymbol{p}_{\perp}^{o}, \boldsymbol{x}_{\perp}^{o}, t_{o}\right)=t_{0}+\int_{0}^{z} \frac{1}{v_{z}\left(z^{\prime} ; \boldsymbol{p}_{\perp}^{o}, \boldsymbol{x}_{\perp}^{\prime}, t_{0}\right)} d z^{\prime} \\
& \Omega_{4} \equiv\left(\frac{x_{0}}{\Xi_{0}}\right)^{2}+\left(\frac{y_{0}}{\Psi_{0}}\right)^{2}+\left(\frac{p_{x o}}{\Pi_{x o}}\right)^{2} \\
& +\left(\frac{p_{y o}}{\Pi_{y o}}\right)^{2} \leq 1
\end{aligned}
$$

where $\Omega_{4}$ denotes the hypervolume in phase space with boundaries $\Xi_{o}, \Psi_{o}, \Pi_{x o}$, and $\Pi_{y o} ; T$ is the average transit time of an electron through the interaction region; $D\left(\boldsymbol{p}_{\perp}^{\circ}\right.$, $\boldsymbol{x}_{\perp}^{\prime \prime}$ ) is the distribution function over the hypervolume $\Omega_{4}$; and $\sigma_{\|}\left(t_{o}\right)$ is the entrance-time distribution. The notation $\tau$ is the time when an electron, entering at $t_{o}$, arrives at the axial position $z$. The distribution functions are subject to the following normalizations:

$$
\begin{aligned}
& \iiint_{\Omega_{4}} \int_{d o} d p_{x o} d p_{y o} d x_{o} d y_{o} D\left(p_{\perp}^{o}, \boldsymbol{x}_{\perp}^{\prime \prime}\right)=1 \\
& \int_{-T / 2}^{T / 2} d t_{o} \sigma_{\|}\left(t_{o}\right)=T .
\end{aligned}
$$

The hypervolume $\Omega_{4}$ has the shape of a hyper-ellipsoid, and its volume can be used as a figure-of-merit of beam quality, such as the normalized four-volume emittance $\hat{\epsilon}_{4}$ $=V_{4}=\frac{1}{2} \Xi_{0} \Psi_{0} \Pi_{3 o} \Pi_{10}[16]$ and the brightness $B_{N}=I_{b} / V_{4}$ [17]. Furthermore, $\dot{\hat{\epsilon}}_{4}$ can be written as the product of $x$-plane and $y$-plane normalized root-mean-square emittances, i.e., $\hat{\epsilon}_{4}=\frac{1}{2} \hat{\epsilon}_{x(\mathrm{rm}) \mathrm{s})} \hat{\epsilon}_{\mathrm{v}(\mathrm{rm}) \mathrm{s})}$, where the root-meansquare emittance is defined by Fraser et al. [18]. Therefore we obtain the following results. For a Gaussian distribution.

$$
\begin{aligned}
& \hat{\epsilon}_{x(\mathrm{rm}))}=2 \pi \Xi_{0} \Pi_{x, 1}, \quad \hat{\epsilon}_{y(\mathrm{~mm})}=2 \pi \Psi_{0} \Pi_{\left.y^{\prime}\right)} \\
& B_{N}=8 \frac{I_{b}}{\hat{\epsilon}_{x(\mathrm{mms})} \hat{\epsilon}_{v(\mathrm{mms})}} .
\end{aligned}
$$

For a uniform distribution,

$$
\begin{aligned}
\hat{\epsilon}_{x(\mathrm{~m} m \mathrm{~s})} & =\frac{2}{3} \pi \Xi_{o} \Pi_{w o}, \quad \hat{\epsilon}_{y(\mathrm{mms})}=\frac{2}{3} \pi \Psi_{o} \Pi_{w o} \\
B_{N} & =\frac{8}{9} \frac{I_{b}}{\hat{\epsilon}_{x(\mathrm{mms})} \hat{\epsilon}_{y(\mathrm{mms})}} .
\end{aligned}
$$

Note that the same emittance will give a factor of nine difference in the value of brightness for the two distributions considered.

The wiggler field is generated by an array of parabolically shaped permanent magnets [19] and is shown to have a second-order focusing effect such that it enhances the FEL performance over the conventional linear dipole. We allow variation of $\boldsymbol{B}_{w},(z)$ along the $z$ axis for the study of efficiency enhancement. According to the KMR paper [11], the taper rate satisfying the equation

$$
\frac{d a_{w}}{d z}=-2 k_{n} \cdot a_{\mathrm{ln}}(z) \sin \Psi_{\mathrm{res}}
$$

ensures that the $\ln$ th ponderomotive wave stays resonant with the slowing electron beam, where $a_{w}$ and $a_{l n}$ represent the normalized vector potential of the wiggler and the Inth waveguide mode field, respectively. In addition, the KMR nonlinear wiggler taper is able to reduce unwanted competing modes, since each competing wave propagates by its own phase velocity, so that each ponderomotive wave has its own optimal taper rate.

If we assume that the space-charge force is weak compared with the ponderomotive force, the radiation field can be expanded in terms of the vacuum waveguide modes; i.e.,

$$
\begin{aligned}
\boldsymbol{A}(\boldsymbol{x}, t)= & \sum_{\mathrm{TE} \ln } A_{\ln }^{\mathrm{TE}}(z) \cos \alpha_{\ln }(z, t) \hat{\boldsymbol{e}}_{\ln }^{1}(x, y) \\
& +\sum_{\mathrm{TM}_{\ln }} A_{\ln }^{\mathrm{TM}}(z)\left(\cos \alpha_{\ln } \hat{\boldsymbol{e}}_{\mathrm{ln}}^{2}(x, y)\right. \\
& \left.+\sin \alpha_{\ln } \hat{\boldsymbol{e}}_{\ln }^{3}(x, y)\right) .
\end{aligned}
$$

The notation

$$
\alpha_{\ln }(z, t) \equiv\left(\int_{0}^{\sigma} k_{\ln }\left(z^{\prime}\right) d z^{\prime}-\omega t\right)
$$


represents the phase of waveguide mode, and $\hat{\boldsymbol{e}}_{1 \mathrm{n}}(x, y)$, $\hat{\boldsymbol{e}}_{\mathrm{ln}}^{2}(x, y)$ and $\hat{\boldsymbol{e}}_{\mathrm{ln}}^{3}(x, y)$ are mode functions, defined as follows:

$$
\begin{aligned}
\hat{\boldsymbol{e}}_{\ln }^{1}(x, y)= & \frac{k y}{k_{c \cdot \ln }} \cos k_{x}\left(x+\frac{a}{2}\right) \sin k_{y}\left(y+\frac{b}{2}\right) \hat{\boldsymbol{x}} \\
& -\frac{k_{x}}{k_{c \cdot \ln }} \sin k_{x}\left(x+\frac{a}{2}\right) \cos k_{y}\left(y+\frac{b}{2}\right) \hat{\boldsymbol{y}} \\
\hat{\boldsymbol{e}}_{\mathrm{ln}}^{2}(x, y)= & \frac{k x}{k_{c \cdot \ln }} \cos k_{x}\left(x+\frac{a}{2}\right) \sin k_{y}\left(y+\frac{b}{2}\right) \hat{\boldsymbol{x}} \\
& +\frac{k_{y}}{k_{c \cdot \ln }} \sin k_{x}\left(x+\frac{a}{2}\right) \cos k_{y}\left(y+\frac{b}{2}\right) \hat{\boldsymbol{y}} \\
\hat{\boldsymbol{e}}_{\ln }^{3}(x, y)= & \frac{k_{c \cdot \ln }}{k_{\ln }} \sin k_{x}\left(x+\frac{a}{2}\right) \sin k_{y}\left(y+\frac{b}{2}\right) \hat{z}
\end{aligned}
$$

where

$$
k_{x}=\frac{l \pi}{a}, \quad k_{y}=\frac{n \pi}{b}, \quad k_{c \cdot \ln }^{2}=k_{x}^{2}+k_{y}^{2}
$$

( $a$ and $b$ are the width and height of a rectangular waveguide), and $k_{\ln }(z)$ is the wave number of the $\ln$ th mode.

We now can solve the inhomogeneous wave equation by substituting the expression of the vector potential $\boldsymbol{A}(\boldsymbol{x}$, $t)$ and source current density $\boldsymbol{J}(\boldsymbol{x}, t)$ and by utilizing the orthonormality properties. As a result, we obtain the following set of equations, which are identical to those derived in the Freund et al. paper [6] for the TE mode:

$$
\begin{aligned}
& \frac{d^{2}}{d z^{2}} a_{\ln }+\left(\frac{\omega^{2}}{c^{2}}-k_{\ln }^{2}-k_{c, \ln }^{2}\right) a_{\ln } \\
& =2 \frac{\omega_{b}^{2}}{c^{2}} H_{\ln }\left\langle\hat{\boldsymbol{e}}_{\ln }^{1}(x, y) \cdot \frac{v}{\left|v_{z}\right|} \cos \alpha_{\ln }\right\rangle \\
& 2 \sqrt{k_{\ln }} \frac{d}{d z}\left(\sqrt{k_{\ln }} a_{\ln }\right) \\
& =-2 \frac{\omega_{b}^{2}}{c^{2}} H_{\ln }\left\langle\hat{\boldsymbol{e}}_{\ln }^{1}(x, y) \cdot \frac{v}{\left|v_{z}\right|} \sin \alpha_{\ln }\right\rangle .
\end{aligned}
$$

For the TM mode,

$$
\begin{aligned}
\frac{d^{2}}{d z^{2}} a_{\ln } & +\left(1+\frac{k_{c, \ln }^{2}}{k_{\ln }^{2}}\right)\left(\frac{\omega^{2}}{c^{2}}-k_{\ln }^{2}-k_{c, \ln }^{2}\right) a_{\ln } \\
= & 2 \frac{\omega_{b}^{2}}{c^{2}} H_{\ln } \\
& \times \quad \hat{\boldsymbol{e}}_{\ln }^{2}(x, y) \cdot \frac{v}{\left|v_{*}\right|} \cos \alpha_{\ln }+\hat{\boldsymbol{e}}_{\ln }^{3}(x, y) \\
& \cdot \frac{v}{\left|v_{z}\right|} \sin \alpha_{\ln }
\end{aligned}
$$

$$
\begin{aligned}
2 \sqrt{k_{\ln }\left(1+\frac{k_{c \cdot \ln }^{2}}{k_{\ln }^{2}}\right)} \frac{d}{d z}\left(\sqrt{k_{\ln }\left(1+\frac{k_{c \cdot \ln }^{2}}{k_{\ln }^{2}}\right)} a_{\ln }\right) \\
=-2 \frac{\omega_{b}^{2}}{c^{2}} H_{l n} \\
\times \hat{\boldsymbol{e}}_{\ln }^{2}(x, y) \cdot \frac{v}{\left|v_{z}\right|} \sin \alpha_{\ln }-\hat{\boldsymbol{e}}_{\ln }^{3}(x, y) \\
\cdot \frac{v}{\left|v_{z}\right|} \cos \alpha_{\ln }
\end{aligned}
$$

where

$$
a_{\ln }\left(\equiv \frac{e A_{\ln }}{m_{0} c^{2}}\right)
$$

is the normalized mode potential,

$$
\omega_{b}\left(\equiv \sqrt{\frac{4 \pi e^{2} n_{b}}{m_{o}}}\right)
$$

is the nonrelativistic plasma frequency, and

$$
H_{\ln }= \begin{cases}2, & l=0 \text { or } n=0 \\ 4, & \text { otherwise. }\end{cases}
$$

The average operator $\langle\cdots\rangle$ is defined as integration over the hypervolume $\Omega_{4}$ and a full period of ponderomotive phase:

$$
\begin{aligned}
\langle\cdots\rangle \equiv \iiint_{\Omega 4} \int d p_{x o} d p_{y o} d x_{o} d y_{o} D\left(p_{\perp}^{\mathrm{o}}, \boldsymbol{x}_{\perp}^{\mathrm{o}}\right) \\
\cdot \int_{-\pi}^{\pi} d \psi_{o} \sigma_{\|}\left(\psi_{o}\right)(\cdots) .
\end{aligned}
$$

The effect of electron beam source distribution is considered to be incorporated in the average operator.

To describe the electrons' motion, we need to solve the relativistic equations of motion for each electron, including the forces from the wiggler and waveguide fields (indexed by $m$ ), i.e.,

$$
\begin{gathered}
\frac{d \boldsymbol{p}_{i}}{d t}=-e \sum_{m}\left(\boldsymbol{E}_{m}+\frac{\boldsymbol{v}_{i}}{c} \times \boldsymbol{B}_{m}\right)-e \frac{\boldsymbol{v}_{i}}{c} \times \boldsymbol{B}_{\mathrm{w}} \\
i=1,2, \cdots, N_{\mathrm{part}} .
\end{gathered}
$$

Eventually, we obtain the following equations (omitting the index $i$ for clarity):

$$
\begin{aligned}
v_{z} \frac{d}{d z} p_{x}= & \frac{1}{\gamma} k_{\mathrm{u}} c \sqrt{2} a_{\mathrm{w}}\left[\left(\sqrt{2} W_{3} \sin k_{\mathrm{w}} z\right) p_{y}\right. \\
& \left.+\left(W_{2} \cos k_{\mathrm{u}} z\right) p_{z}\right] \\
& +\sum_{\mathrm{TE} \ln } m_{o} c a_{\ln }\left[\left(\omega-k_{\ln } v_{z}\right) \frac{k_{y}}{k_{c \cdot \ln }} B_{2} \sin \alpha_{\ln }\right. \\
& \left.+v_{y} k_{c \cdot \ln } B_{3} \cos \alpha_{\ln }+\Gamma_{\ln } v_{z} \frac{k_{y}}{k_{c \cdot \ln }} B_{2} \cos \alpha_{\ln }\right]
\end{aligned}
$$




$$
\begin{aligned}
v_{*} \frac{d}{d z} p_{x}= & +\sum_{\tau \mathrm{M}_{\ln }} m_{o} c a_{\ln }\left\{\left[\omega-k_{\ln }\left(1+\frac{k_{c \cdot \ln }^{2}}{k_{\ln }^{2}}\right) v_{z}\right]\right. \\
& \left.\cdot \frac{k_{x}}{k_{c \cdot \ln }} B_{2} \sin \alpha_{\ln }+\Gamma_{\ln } v_{x} \frac{k_{x}}{k_{c \cdot \ln }} B_{2} \cos \alpha_{\ln }\right\}
\end{aligned}
$$

$$
\begin{aligned}
& v_{z} \frac{d}{d z} p_{y}=-\frac{1}{\gamma} k_{n} c \sqrt{2} a_{n}\left[\left(\sqrt{2} W_{3} \sin k_{u} z\right) p_{v}\right. \\
& \left.+\left(W_{1} \cos k_{11} z\right) p_{-}\right] \\
& +\sum_{\mathrm{TE} \mid \mathrm{n}} m_{o} c a_{\ln }\left[\left(\omega-k_{\ln } v_{z}\right) \frac{k_{x}}{k_{c \cdot \ln }} B_{1} \sin \alpha_{\mathrm{ln}}\right. \\
& \left.+v_{x} k_{t \cdot \ln } B_{3} \cos \alpha_{\ln }+\Gamma_{\ln } v^{\prime}=\frac{k_{x}}{k_{c \cdot \ln }} B_{1} \cos \alpha_{\ln }\right] \\
& +\sum_{\mathrm{TM}} m_{\mathrm{ln}} c a_{\ln }\left\{\left[\omega-k_{\ln }\left(1+\frac{k_{c \cdot \ln }^{2}}{k_{\ln }^{2}}\right) v_{z}\right]\right. \\
& \left.\cdot \frac{k_{y}}{k_{c \cdot \ln }} B_{1} \sin \alpha_{\mathrm{ln}}+\Gamma_{\ln } v_{z} \frac{k_{y}}{k_{c, \ln }} B_{1} \cos \alpha_{\ln }\right\}
\end{aligned}
$$

$$
\begin{aligned}
& v_{z} \frac{d}{d z} p_{z}=-\frac{1}{\gamma} k_{w} c \sqrt{2} a_{n}\left[W_{2} p_{. r}-W_{1} p_{y}\right] \cos k_{w} \cdot z \\
& +\sum_{\mathrm{TE} E_{\mathrm{ln}}} m_{o} c a_{\ln }\left(v_{x} k_{y} B_{2}-v_{y} k_{x} B_{1}\right) \\
& \cdot\left(\frac{k_{\ln }}{k_{c, \ln }} \sin \alpha_{\ln }-\frac{\Gamma_{\ln }}{k_{c, \ln }} \cos \alpha_{\ln }\right) \\
& -\sum_{\mathrm{TM} \ln } m_{o} c a_{\ln }\left\{\omega \frac{k_{c \cdot \ln }}{k_{\ln }} B_{4} \cos \alpha_{\ln }\right. \\
& -\left(v_{x} k_{x} B_{2}+v_{y} k_{y} B_{1}\right) \\
& \times\left[\frac{k_{\ln }}{k_{c, \ln }}\left(1+\frac{k_{c, \ln }^{2}}{k_{\ln }^{2}}\right) \sin \alpha_{\ln }\right. \\
& \left.\left.-\frac{\Gamma_{\ln }}{k_{c, \ln }} \cos \alpha_{\ln }\right]\right\}
\end{aligned}
$$

where

$$
a_{\mathrm{H}}(z)\left(\equiv \frac{e B_{w}(z)}{\sqrt{2} m_{o} c^{2} k_{w}}\right)
$$

is the normalized wiggler potential;

$$
\Gamma_{\ln }\left(\equiv \frac{d}{d z} \log _{e} a_{\ln }(z)\right)
$$

denotes the growth rate of the respective waveguide modes; $B_{1}(x, y), B_{2}(x, y), B_{3}(x, y), B_{4}(x, y)$ are functions related to the transverse variation of the waveguide modes; and $W_{1}(x, y), W_{2}(x, y)$, and $W_{3}(x, y)$ describe the transverse dependence of the wiggler field as follows:

$$
\begin{aligned}
& B_{1}(x, y)=\sin k_{x}\left(x+\frac{a}{2}\right) \cos k_{y}\left(y+\frac{b}{2}\right) \\
& B_{2}(x, y)=\cos k_{x}\left(x+\frac{a}{2}\right) \sin k_{y}\left(y+\frac{b}{2}\right) \\
& B_{3}(x, y)=\cos k_{x}\left(x+\frac{a}{2}\right) \cos k_{y}\left(y+\frac{b}{2}\right) \\
& B_{4}(x, y)=\sin k_{x}\left(x+\frac{a}{2}\right) \sin k_{y}\left(y+\frac{b}{2}\right) \\
& W_{1}(x, y)=\sinh \left(\frac{k_{11} x}{\sqrt{2}}\right) \sinh \left(\frac{k_{11} y}{\sqrt{2}}\right) \\
& W_{2}(x, y)=\cosh \left(\frac{k_{w} x}{\sqrt{2}}\right) \cosh \left(\frac{k_{11} y}{\sqrt{2}}\right) \\
& W_{3}(x, y)=\cosh \left(\frac{k_{1 x} x}{\sqrt{2}}\right) \sinh \left(\frac{k_{11} y}{\sqrt{2}}\right) .
\end{aligned}
$$

We then expand the equation $\boldsymbol{v}=d \boldsymbol{x} / d t$, and write the ponderomotive phase evolution for $\Psi_{l n}(z)$ for the ith electron with respect to the $\ln$ th waveguide mode. Equations (5), (6), and (9)-(11) constitute a set of coupled differential equations, which describes the wave-particle interaction in a 3-D manner and includes effects of mode competition, wiggler tapering, and beam source distributions.

\section{Simulation Results at $280 \mathrm{GHz}$}

We have developed a 3-D simulation code that consists of a beam-wave loader, a wave-particle solver, and a postprocessor. In the beam-wave loader, we launch the waveguide modes with given power levels and prepare electrons with assigned beam brightness and distribution functions. Since we use the $N$ th-order Gaussian quadrature technique to evaluate the average operator, electrons are loaded with coordinates and momenta specified by the technique itself. In the wave-particle solver, the fourthorder Runge-Kutta-Gill method is applied for solving the set of coupled differential equations. The numerical error for this algorithm is proportional to the fifth power of the step size. The post-processor then documents the simulation results with optional graphics.

Our version of the 3-D code has two different algorithms as compared with those of the Freund et al. 3-D code [6], [7]. First, the electron beam source in [6] and [7] has uncorrelated momenta and coordinate distribution functions, which is most suitable for the thermionic electron sources. However, for the induction linacs considered in this paper, as well as RF-linacs, the distributions of electron-particle momenta and coordinates are correlated and are more appropriately modeled by an aggregate distribution function over momenta and coordinate space. Second, our 3-D code allows the wiggler amplitude profile to be tapered nonlinearly as well as the linear taper that is considered in the previous works [6], [7]. 
Our 3-D code also differs when compared with the wiggler-averaged WFEL code [20]. First of all, the WFEL code simulates only the slow time-scale mechanisms for the average over a wiggler period, eliminating the fast timescale variation; our 3-D code keeps both timescale variations. Second, the inclusion of mode competition in the WFEL code is via the calculation of mode-coupling coefficients with explicit analytic expressions [20]; our 3 -D code includes mode competition implicitly through (5)-(6) and the equations of motion (9)-(12). The electric space-charge field in the WFEL code is calculated analytically by treating the electron beam as a wiggling fluid flow and solving the wave equation subject to modified boundary conditions. To simplify the algebraic calculation, the size of electron beam is assumed to be much smaller than the waveguide size, and the average process with the FEL synchronism condition satisfied is utilized.

A simulation of an FEL amplifier operating at $280 \mathrm{GHz}$ has been carried out with the following parameters: $V_{b}=$ $10 \mathrm{MeV}, I_{b}=3000 \mathrm{~A}, r_{b}=0.4537 \mathrm{~cm}, \epsilon=251 \mathrm{mrd} \cdot$ $\mathrm{mm}, B_{N}=1 \times 10^{4} \mathrm{~A} /(\mathrm{rad} \cdot \mathrm{cm})^{2}$ with $B_{11}=4.09 \mathrm{kG}$, $\lambda_{w}=10 \mathrm{~cm}$, and a guide which is $3.10 \mathrm{~cm}^{2}$. The $\mathrm{TE}_{01}$ mode is injected at $458 \mathrm{~W}$ with residual $\mathrm{TM}_{21}, \mathrm{TE}_{21}$, and $\mathrm{TE}_{03}$ modes excited at $65-\mathrm{mW}$ levels, corresponding to the anticipated values for the planned Livermore FEL experiment [2]. This FEL is operated in the transition between the Compton and the Raman regimes, or the marginal Raman regime, when a comparison of ponderomotive and space-charge forces and length of the wiggler are considered. For the uniform wiggler case, as shown in Fig. 2, the total saturation power is $1.4 \mathrm{GW}$ at $k_{11} z=142(z=2.16 \mathrm{~m})$, which corresponds to a growth rate $30 \mathrm{~dB} / \mathrm{m}$ or an efficiency of 4.6 percent. The $\mathrm{TM}_{21}$ and $\mathrm{TE}_{21}$ modes are shown to compete substantially with the $\mathrm{TE}_{01}$ mode, reaching 50 percent of the wave power near $k_{11}, z=50$.

We then add a nonlinear taper section for efficiency enhancement. As pointed out by Jong et al. [3], the optimal beginning taper position is not solely determined by maximizing the $\mathrm{TE}_{0}$ power at the end of the constant section but, more importantly, by considering energy and phase spread within the ponderomotive potential well. This is because significant energy spread will prevent electrons from remaining trapped in the ponderomotive well. Fig. 3 shows electron evolution in $(\gamma, \psi)$ space at different axial positions and indicates that $k_{w} z=115$ is the optimal tapering position according to preceding argument. On the other hand, the beam confinement by the wiggler is proportional to the wiggler's magnitude, so that we terminate the tapering before the electron beam blows up and intersects the wall. As a result, the efficiency is enhanced to 48 percent and the higher-order modes considered are reduced to less than 2 percent, as shown in Fig. 4 . In other words, there is $14.1 \mathrm{GW}$ in the $\mathrm{TE}_{01}$ mode together with $0.3 \mathrm{GW}$ in higher-order modes at the end of wiggler. We also examined a linear taper with the rate $d a_{w} / d z=$ $-0.10 \mathrm{~cm}^{-1}$ and found that higher-order modes contain up to 10 percent of the total wave power with the effi-

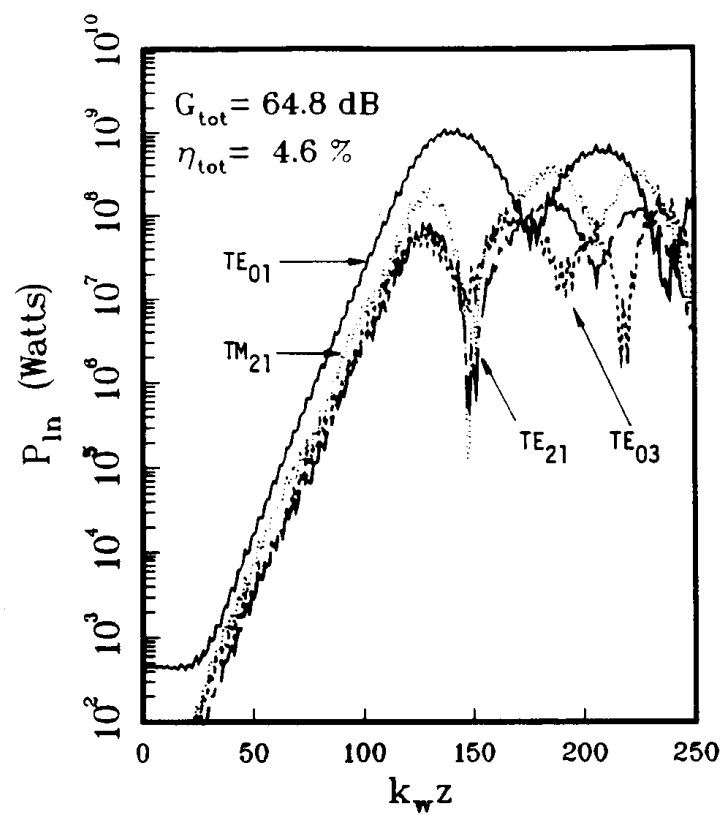

Fig. 2. Solution of $280-\mathrm{GHz}$ mode power for untapered wiggler.

ciency reduced to 42 percent, as compared with a total higher-order mode power of 2 percent with an efficiency of 48 percent for the nonlinear taper case. This shows the superiority of the KMR nonlinear taper to the linear taper for efficiency enhancement and higher-order mode suppression. We also looked at the sensitivity of the $\mathrm{TE}_{01}$ power to the assumed higher-order-mode input power levels. We found that, at higher power levels, they can influence the $\mathrm{TE}_{01}$ power evolution, as is evident in Fig. 5 . Note that the $\mathrm{TE}_{01}$ mode damps initially and then grows normally when higher-order mode power is increased up to $65 \mathrm{~W}$ (three orders of magnitude). Nevertheless, the $\mathrm{TE}_{01}$ power is reduced by only 6 percent at the end of the wiggler (cf. the case for Fig. 4).

As mentioned in the preceding, we neglect space-charge forces in the 3-D code. To address the effects of the spacecharge force, we compare the results with those from the WFEL code. The WFEL code, developed by Byers and Cohen [20], is a wiggler-averaged simulation that includes space-charge forces. It has been shown to have excellent agreement with the 35-GHz FEL experiment at the Lawrence Livermore National Laboratory. In Fig. 6, our 3-D results, compared with those from the WFEL code without space-charge forces, show that the growth rate during the initial constant wiggler section and in the taper region are slightly different. Due to this difference, and the constraint that the taper must continue down to a field of $600 \mathrm{G}$, the total interaction lengths differ by 10 percent ( $3.6 \mathrm{~m}$ for the 3 -D code versus $4.0 \mathrm{~m}$ for the WFEL code). This indicates that there are some slight differences between the 3-D and wiggler-averaged formulations. If space-charge forces are included in the WFEL code, we find that the growth rate both before and within 


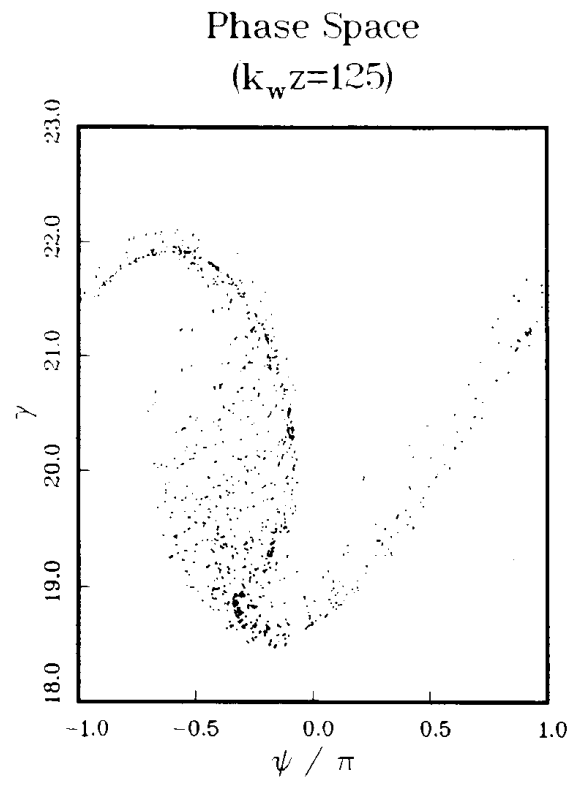

(a)

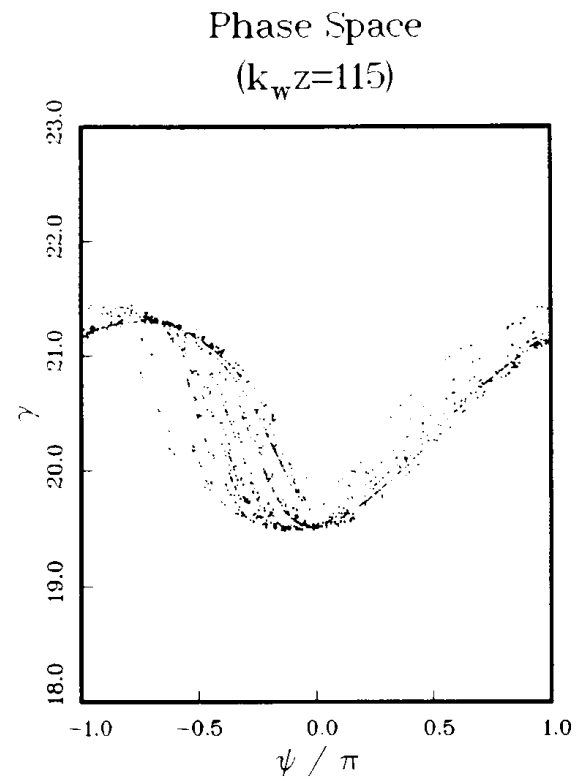

(b)

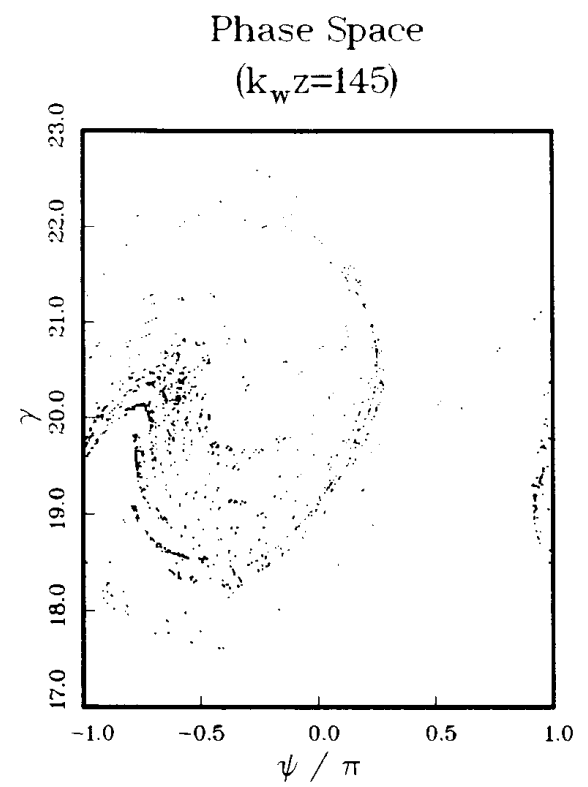

(c)

Fig. 3. Phase space $(\gamma, \psi)$ evolution of electron beam. (a) At $k_{w} z=115$. (b) At $k_{11} z=125$. (c) At $k_{11} z=145$.

the taper section is significantly reduced. This results in reducing the efficiency to 32 percent as well as changing the wiggler taper profile.

To compare our works with others, we examine the results discussed in the paper by Ganguly and Freund [10]. The closest they come to the case discussed in this paper is a $1-\mathrm{MeV} 50-\mathrm{A}$ case for which the efficiency reduction due to space charge is typically under 5 percent at a frequency near $35 \mathrm{GHz}$. The addition of the strong guide field, shorter total wiggler length, lower beam current, and lower operating frequency for their case is the reason for a 5-percent reduction in efficiency as compared to the 16-percent reduction in efficiency for our case.

We also compare the results of Fig. 6 with those obtained using the FRED code discussed in [2] and [3]. Comparison with [2, fig. 6] and [3, fig. 4] shows efficiencies from 37 to 33 percent for $6 \mathrm{~m}\left(B=10^{5} \mathrm{~A} /(\mathrm{rad}-\mathrm{cm})^{2}\right)$ and $4.6 \mathrm{~m}\left(B=10^{4} \mathrm{~A} /(\mathrm{rad}-\mathrm{cm})^{2}\right)$, respectively. For our 


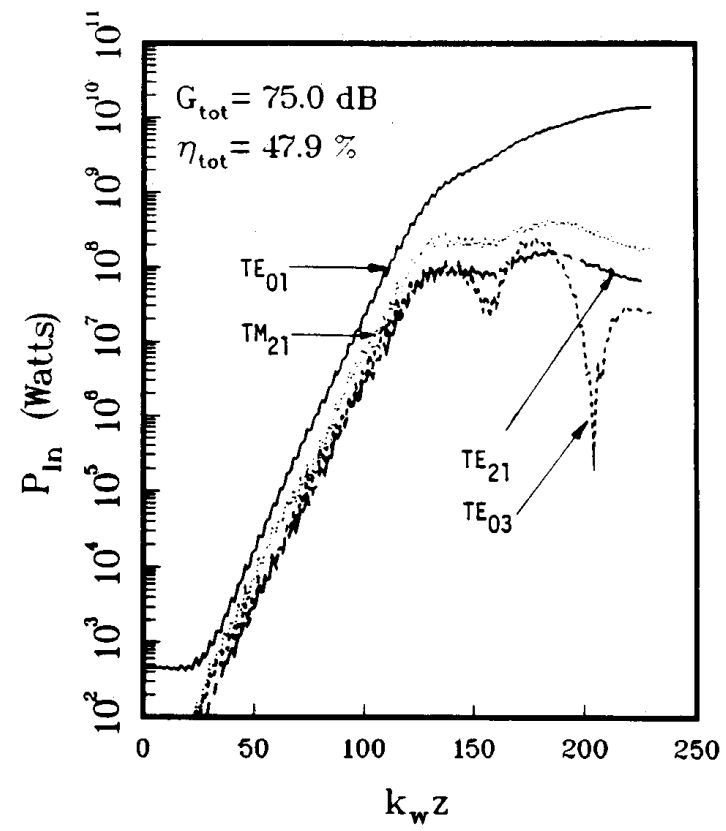

Fig. 4. Evolution of $280-\mathrm{GHz}$ mode power for nonlinear taper.

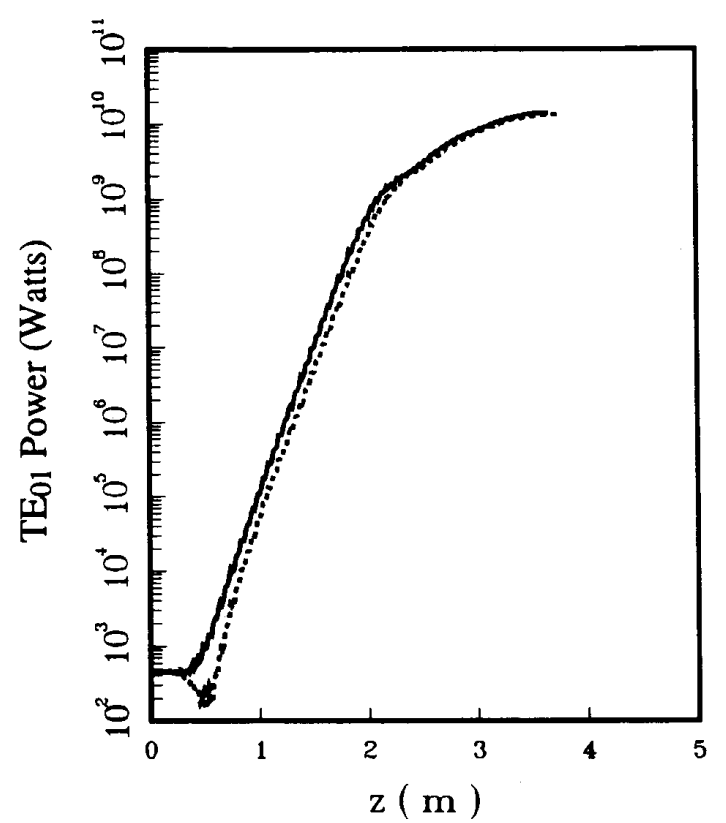

Fig. 5. Evolution of $\mathrm{TE}_{4 \prime \prime}$ power with higher-order mode input power at $68 \mathrm{~mW}$ each (solid line) and at $68 \mathrm{~W}$ each (dashed line).

comparable case, the efficiency is 32 percent for a wiggler of 4-m length with a beam brightness of $10^{4} \mathrm{~A} /(\mathrm{rad}$. $\mathrm{cm})^{2}$. Although the exact same cases were not run for both codes, the code results appear comparable. Our results yield a higher efficiency per unit wiggler length due to our use of a $0.55-\mathrm{rad}$ psi resonant (which determines

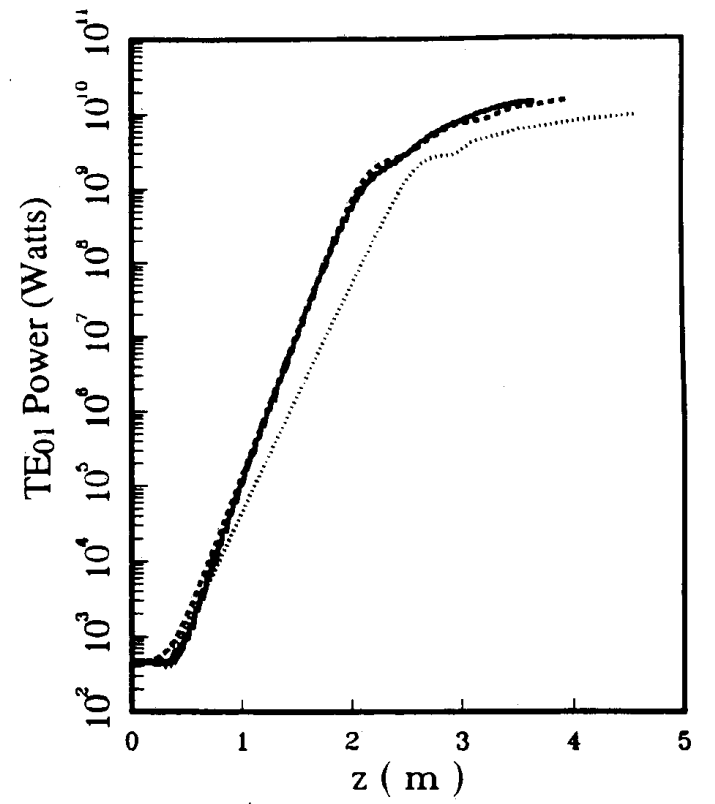

Fig. 6. Comparison of 3-D solution (solid line) and solutions from wig gler-averaged code without (dashed line) and with (dotted line) spacecharge forces.

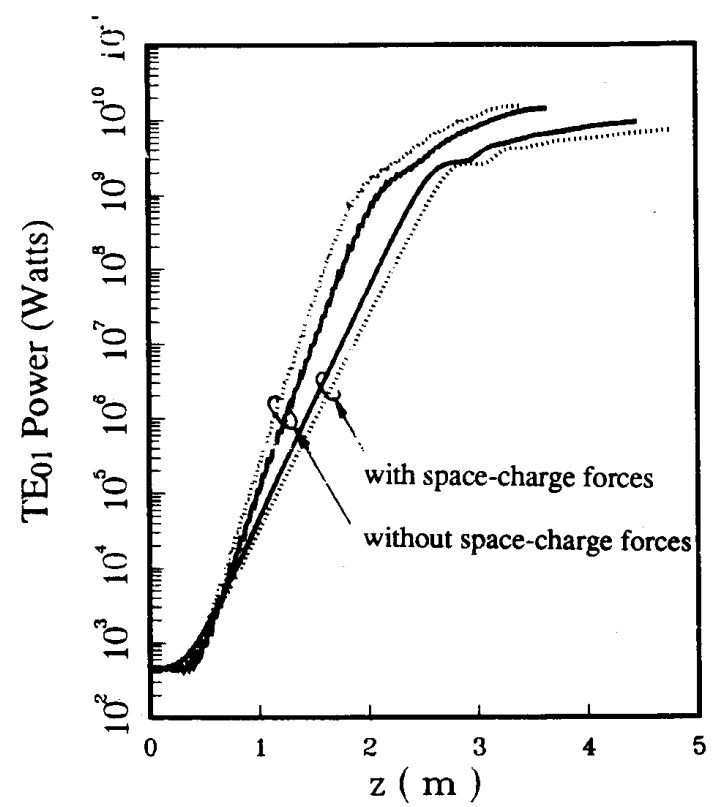

Fig. 7. Comparison of uniform (solid line) and Gaussian (dotted line) source distributions without and with space-charge forces.

the taper properties) rather than that of $0.1-0.2 \mathrm{rad}$, which was used for their cases.

We also investigated the effect of electron beams with different source distributions over the regimes from small signal to saturation in the millimeter wave regime. Two cases are examined: one is uniform distribution and the 
other is Gaussian distribution. First, we look at the condition with the same brightness: $1 \times 10^{4} \mathrm{~A} /(\mathrm{rad} \cdot \mathrm{cm})^{2}$ (emittance is changed following (2)). We find that, in both cases, the growth rate and the power evolution are almost the same. Second, we fix the emittance at $251 \mathrm{mrd} \cdot \mathrm{mm}$ and change the brightness according to (2), which makes the brightness $1 \times 10^{4} \mathrm{~A} /(\mathrm{rad} \cdot \mathrm{cm})^{2}$ for the uniform distribution and $9 \times 10^{4} \mathrm{~A} /(\mathrm{rad} \cdot \mathrm{cm})^{2}$ for the Gaussian distribution. Fig. 7 shows that, without the inclusion of space-charge forces, the growth rate of the Gaussian distribution is larger than that of the uniform. In addition, the $15.3 \mathrm{GW}$ of $\mathrm{TE}_{01}$ output power for the Gaussian distribution is 8.5-percent larger than the $14.1 \mathrm{GW}$ for the uniform case. Note that different optimal taper positions are chosen for each case. If space-charge forces are included, the $\mathrm{TE}_{01}$ output power of the Gaussian distribution is reduced even further compared to that of the uniform case. Qualitatively, the brighter beam allows a smaller beam radius to carry the same amount of current, so that space-charge forces become stronger and reduce the efficiency further.

\section{Conclusion}

We have carried out a 3-D simulation of an FEL amplifier at $280 \mathrm{GHz}$, including effects of wiggler tapering, mode competition, and different beam source distributions. The nonlinear wiggler taper has been shown to be superior to the linear variety in terms of efficiency enhancement and unwanted mode suppression. Our 3-D results agree very well with those from the wiggler averaged code when space-charge effects are neglected; moreover, we find space-charge forces reduce the efficiency from 48 percent down to a level of 32 percent for a $10-\mathrm{MeV} 3-\mathrm{kA}$ beam and $10-\mathrm{cm}$ period linear dipole wiggler.

The sensitivity of FEL gain to higher-order mode power and electron beam source distributions is examined as well. We infer that higher-order-mode power levels varying over three orders of magnitude can affect the $\mathrm{TE}_{01}$ power evolution but only slightly reduce the $\mathrm{TE}_{01}$ output power. As to the effect of beam source distributions, we find that only distributions with same emittance have observable differences. In the Compton regime, an electron beam with Gaussian distribution gives a higher growth rate and higher $\mathrm{TE}_{01}$ output power; however, the uniform distribution has larger growth rate and $\mathrm{TE}_{01}$ output power if space-charge forces are included.

\section{REFERENCES}

[1] T. J. Orzechowski, B. R. Anderson, et al., "High gain and high extraction efficiency from a free electron laser amplifier operating in the millimeter wave regime," Nucl. Instrum. Methods Phys. Res., vol. A250, pp. 144-189, 1986.

[2] R. A. Jong and E. T. Scharlemann, "High gain free electron laser for heating and current drive in the Alcator-C Tokamak," Nucl. Instrum. Methods Phys. Res., vol. A259, pp. 254-258, 1987

[3] R. A. Jong, A. L. Throop, and E. T. Scharlemann, "Baseline design for the IMP wiggler," Rev. Sci. Instrum., vol. 60, p. 186, 1989.

[4] A. K. Ganguly and H. P. Freund, "Nonlinear analysis of free-electron-laser amplifiers in three dimensions," Phys. Rev. A, vol. 32, no. 4, pp. 2275-2286, Oct. 1985.
[5] H. P. Freund and A. K. Ganguly, "Nonlinear analysis of efficiency enhancement in free-electron-laser amplifiers," Phys. Rev. A, vol. 33, no. 2, pp. 1060-1072, Feb. 1986.

[6] H. P. Freund, H. Bluem, and C. L. Chang, "Three-dimensional nonlinear analysis of free-electron-laser amplifiers with planar wigglers,"' Phys. Rev. A, vol. 36, no. 5, pp. 2182-2198, Sept. 1987.

[7] H. P. Freund, "Multi-mode nonlinear analysis of free-electron-laser amplifier in three-dimension," Phys. Rev. A, vol. 37, no. 9, pp. 3371$3380,1988$.

[8] J. E. Scharer, O. C. Eldridge, S. F. Chang, and N. T. Lam, "Nonlinear analysis and simulation of a millimeter range free-electron laser with a helical quadrupole wiggler," Nucl. Intrum. Methods Phys. Res., vol. A272, pp. 289-292, Nov. 1988.

[9] S. F. Chang, O. C. Eldridge, and J. E. Scharer, "Nonlinear analysis and simulation of millimeterwave FEL amplifiers," IEEE J. Quant. Electron., vol. QE-24, pp. 2308-2320, Nov. 1988.

[10] A. K. Ganguly and H. P. Freund, "Three-dimensional simulation of the Raman free electron laser," Phys. Fluids, vol. 31, pp. 387-393, 1988.

[11] N. M. Kroll, P. L. Morton, and M. N. Rosenbluth, "Free-electron lasers with variable wigglers," IEEE J. Quant. Electron., vol. QE17, no. 8, pp. 1436-1468, Aug. 1981 .

[12] R. A. Cover and A. Bhowmik, "Enhanced performance from highextraction-efficiency free electron lasers," Nucl. Instrum. Methods Phys. Res., vol. A272, pp. 117-119, Nov. 1988.

[13] J. C. Goldstein, B. D. McVey, and B. E. Newnam, "Gain physics of RF-linac-driven XUV free-electron lasers," Amer. Inst. Phys., vol. 147, pp. 275-290, 1986.

[14] W. B. Colson and J. Blau, "Free electron laser theory in weak optical fields," Nucl. Instrum. Methods Phys. Res., vol. A259, pp. 198-202, 1987.

[15] J. K. Boyd, W. B. Colson, and E. T. Scharlemann, "Initial electron distributions for free electron lasers generated by injector and accelerator simulation," Nucl. Instrum. Methods Phys. Res., vol. A272, pp. 590-594, 1988

[16] T. R. Walsh, "A normal beam with linear focussing and space-charge forces," J. Nucl. Ener., Part C (Plasma Physics), vol. 5, pp. 17-22. 1963.

[17] A. Van Steenbergen, "Studies of beam behavior in the energy region of $50-400 \mathrm{MeV}$ with the Brookhaven alternating gradient synchrotron," IEEE Trans. Nucl. Sci., vol. NS-14, pp. 641-652, June 1967.

[18] J. S. Fraser, R. L. Sheffield, E. R. Gray, and W. Rodenz, "Highbrightness photoemitter injector for electron accelerators," IEEE Trans. Nucl. Sci., vol. NS-32, no. 5, pp. 1791-1793, Oct. 1985.

[19] E. T. Scharlemann, "Wiggler plane focusing in linear wigglers," $J$. Appl. Phys., vol. 58, no. 6, pp. 2154-2161, Sept. 1985.

[20] J. A. Byers and R. H. Cohen, "A microwave FEL code using waveguide modes," Nucl. Instrum. Methods Phys. Res, , vol. A272, pp. $595-600,1988$.

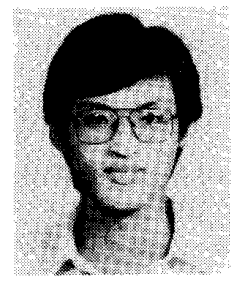

Sheng-Fuh R. Chang was born in Taiwan, Republic of China, in 1960. He received the B.S. and M.S. degrees in communication engineering from the National Chiao-Tung University in 1982 and 1984 , respectively

He has been working towards the Ph.D. degree in electrical and computer engineering and is a member of the Center for Plasma Theory and Computation at the University of Wisconsin, Madison. His research interests are in theoretical and computational investigations in the area of coherent radiation sources, plasma waves, and microstrip circuits. Mr. Chang is a member of Phi Tau Phi. 


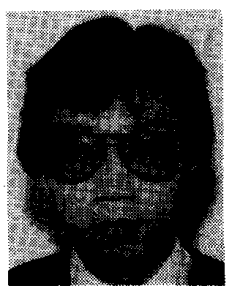

Jurianto Joe ( $S^{\prime} 90$ ) was born in Medan, Indonesia, on July 20,1965 . He received the B.S. degree in electrical engineering from the University of Texas at Arlington in 1988. Presently, he is en gaged in the study of the Cerenkov FEL, working towards the Ph.D. degree at the University of Wisconsin, Madison.

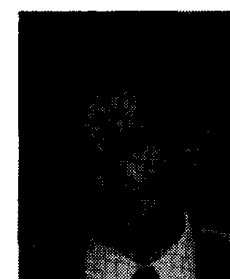

John E. Scharer was born in Monroe, WI, in 1939. He received the B.S., M.S., and Ph.D. degrees in electrical engineering from the University of California, Berkeley, in plasma physics.

$\mathrm{He}$ is a Professor with the Department of Electrical and Computer Engineering, University of Wisconsin, Madison. He has spent research sabatticals at the CEA Fontenay-aux-Roses, France, in 1970 and at the JET tokamak at Culham, England in 1983. He has been active with graduate students and scientists in theoretical, computational, and experimental research in the area of linear and nonlinear plasma waves and heating of fusion plasmas and lasers. He has also taught and recently started research in the area of free-electron lasers and coherent sources of radiation.

Dr. Scharer is a member of Tau Beta Pi, the American Physical Society, and the Center for Plasma Theory and Computation at the University of Wisconsin. 